\title{
Manejo aplicado al sistema porcino tradicional en los llanos colombo-venezolanos
}

\author{
Milena Esney Hernández-Rodríguez ${ }^{\star}, M V Z$, Esp., Luis Ernesto Rodríguez, \\ MV, Esp. ${ }_{1}$, Adolfo F. Cardozo B., Ing. ${ }_{2}$, Arcesio Salamanca C., Zoot.
}

${ }_{1}$ Grupo de Investigaciones Los Araucos, Facultad de Medicina Veterinaria y Zootecnia, Universidad Cooperativa de Colombia, Arauca, Colombia

${ }_{2}$ Centro para la Investigación en Sistemas Sostenibles de Producción Agropecuaria (CENDI),

Universidad de los Llanos Ezequiel Zamora, Guanare, Estado Portuguesa, Venezuela

\begin{abstract}
Recibido: 1 de junio del $2015 \quad$ Aprobado: 12 de diciembre del 2015
*Autor de correspondencia: Milena Esney Hernández. Grupo de Investigaciones Los Araucos, Facultad de Medicina Veterinaria y Zootecnia, Universidad Cooperativa de Colombia. Carrera 12 n. ${ }^{\circ}$ 21-70, Barrio Unión, Arauca, Colombia. Teléfono: (57) 3124319623. Correo electrónico: milena.hernandez@campusucc.edu.co
\end{abstract}

Cómo citar este artículo: Hernández-Rodríguez ME, Rodríguez LE, Cardozo AF, Salamanca A. Manejo aplicado al sistema porcino tradicional en los Ilanos colombo-venezolanos. Spei Domus. 2015;11(23):55-63. doi: http://dx.doi.org/10.16925/ sp.v11i23.1368

Resumen. Propósito: el objetivo del presente artículo fue hacer un análisis del manejo tradicional aplicado a la producción de cerdos criollos en los llanos colombo-venezolanos. Descripción: inicialmente, se describe el origen de los cerdos criollos como un recurso zoogenético que habita en las sabanas colombo-venezolanas, producto de la selección natural. Después, se revela la importancia socioeconómica para los llanos venezolanos de Apure, Guárico y Barinas, y para el oriente de los departamentos colombianos de Casanare, Vichada y Arauca. Se describen algunas de las prácticas de manejo implementadas en este tipo de sistema y se muestran ciertos indicadores productivos. Punto de vista: en general, el cerdo sabanero demuestra una particular independencia frente a los sistemas de producción convencional, razón por la cual ha podido sobrevivir dentro del sistema de explotación tradicional. Esto es un buen ejemplo de la producción limpia e invita a una reflexión que bien vale la pena analizar con más detalle, a fin de ajustar la producción a parámetros comerciales que permitan la continuidad y la supervivencia de este valioso recurso genético local. Conclusiones: se concluye que hay una oportunidad de ofertar productos orgánicos de primera calidad y que, después de esta reflexión acerca del aporte del potencial del cerdo sabanero para satisfacer la demanda de mercados sofisticados y exigentes, se hace necesario desarrollar investigaciones que conduzcan a establecer parámetros productivos y reproductivos que permitan mejorar la eficiencia de este sistema de explotación con una raza porcina subutilizada en el ecosistema de sabanas inundables.

Palabras clave: boral, cerdo criollo, ecosistemas inundables, madrinera, sabana. 


\title{
Management Applied to the Traditional Pig Production System in the Colombian-Venezuelan Plains
}

\begin{abstract}
Purpose: The purpose of this article was to analyze the traditional management applied to the production of native pigs in the Colombian-Venezuelan plains. Description: Initially, the origin of native pigs is described as an animal genetic resource that inhabits the Colombian-Venezuelan savannas, as a result of natural selection. Then, the socio-economic importance to the Venezuelan plains of Apure, Guarico and Barinas, and to the east of the Colombian departments of Casanare, Vichada, and Arauca is revealed. Some of the management practices implemented in this type of system are described and certain production indicators are shown. Point of view: Generally, the savanna pig shows particular independence from conventional production systems and, therefore, it has survived within the traditional exploitation system. This is a good example of clean production and an invitation to reflect which should be analyzed in more detail in order to adjust production to commercial parameters that allow continuity and survival of this valuable local genetic resource. Conclusions: It is concluded that there is an opportunity to offer quality organic products and that, after this reflection on the potential of savanna pig to meet the demand of sophisticated and demanding markets, it is necessary to conduct research in order to establish productive and reproductive parameters that improve the efficiency of this exploitation system with an underused pig race in the flood savanna ecosystem.
\end{abstract}

Keywords: boral, native pig, flood ecosystems, madrinera, savanna.

\section{Manejo aplicado ao sistema suíno tradicional na planície colombo-venezuelana}

Resumo. Propósito: O objetivo deste trabalho foi analisar a gestão tradicional aplicada à produção de suínos crioulos nas planícies colombo-venezuelanas. Descrição: inicialmente, descreve-se a origem dos suínos crioulos como recurso zoogenético que habita na planície colombo-venezuelana, produto da seleção natural. Em seguida, revela-se a importância socioeconômica para a planície venezuelana de Apure, Guárico e Barinas, e para o leste dos estados colombianos de Casanare, Vichada e Arauca. Descrevem-se algumas das práticas de gestão implantadas nesse tipo de sistema e mostram-se certos indicadores produtivos. Ponto de vista: em geral, o suíno da planície mostra uma particular independência dos sistemas de produção convencional, razão pela qual vem podendo sobreviver dentro do sistema de exploração tradicional. Isso é um bom exemplo da produção limpa e convida a uma reflexão que vale a pena analisar com mais detalhe a fim de ajustar a produção a parâmetros comerciais que permitam a continuidade e a sobrevivência desse valioso recurso genético local. Conclusões: conclui-se que há uma oportunidade de ofertar produtos orgânicos de primeira qualidade e que, após essa reflexão sobre a contribuição do potencial do porco de planície para satisfazer a demanda de mercados sofisticados e exigentes, se faz necessário desenvolver pesquisas que levem a estabelecer parâmetros produtivos e reprodutivos que permitam melhorar a eficiência desse sistema de exploração com uma raça suína subutilizada no ecossistema de planícies de inundação (várzeas).

Palavras-chave: boral, porco crioulo, ecossistemas inundáveis, pequena manada, planície. 


\section{Introducción}

La porcinocultura tradicional que se desarrolla en la sabana inundable (llanos de Colombia y Venezuela) consiste en mantener cerdos en el sistema extensivo, sin confinamiento ni dietas ajustadas a planes de manejo propios de la porcicultura industrial que se define eficiente, rentable y competitiva. Muchas veces se obvian las potencialidades y limitaciones propias de las comunidades rurales de esta región, en donde se hace inviable la aplicación de costosas y caprichosas tecnologías modernas e importadas, como galpones con corrales o apriscos, pisos de concreto, dietas a base de alimentos balanceados comerciales e incluso planes sanitarios que son discutibles en la mayoría de los casos.

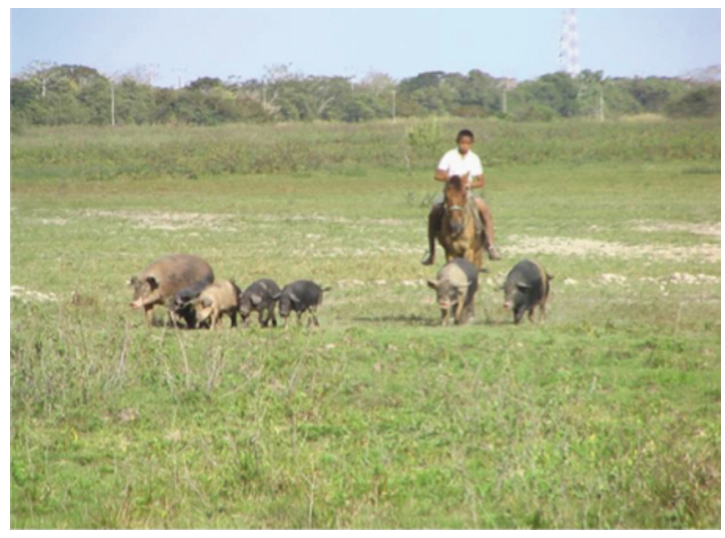

Figura 1. Un muchacho llanero arrea los marranos en la tarde, desde la sabana hasta el corral de la casa

Fuente: Adolfo F. Cardozo, Fundo "San Miguel”, Mantecal, estado Apure, Venezuela

\section{Sistema porcino tradicional}

El marrano sabanero, o chuzo, marrano raudaleño, boraleño, palmareño o pajaleño (dependiendo del sitio donde esté), ha sido de vital importancia en la economía campesina de los llanos colombo-venezolanos, que abarca toda la Orinoquia inundable en los estados venezolanos de Apure, Guárico y Barinas, y en el oriente de los departamentos colombianos de Casanare, Vichada y Arauca. En estos ecosistemas, se ha desarrollado el cerdo criollo sabanero [1], descendiente del cerdo ibérico que llegó a la América tropical hace ya más de quinientos años. Para la Organización de las Naciones Unidas para la
Alimentación y la Agricultura (FAO) y otros autores, se trata de genotipos porcinos locales [2].

No obstante, el cerdo criollo sabanero podría ser considerado una raza naturalizada que se ha adaptado a las más duras condiciones medioambientales del llano inhóspito, donde además del hombre predador, existen los enemigos naturales como la baba o babilla (Crocodilus fuscus o Caimán crocodylus), la culebra de agua o güio (Eunectes murinus), la mapanare de agua (Elicops angulatus), los zamuros o chulos (Coragyps atratus), los caricares o carracos (Caracara plancus), el puma o león barreteado (Felix concolor), el tigre mariposo (Pantera onca), y muchos otros más que se ubican en la cadena trófica y que dependen de la carne que produce esta especie promisoria y extraordinaria de los llanos colombo-venezolanos, especialmente cuando sus presas naturales escasean cada vez más.

La adaptación del cerdo criollo al ecosistema de sabanas inundables ha permitido su aprovechamiento sostenido, pues el poco manejo técnico que demanda lo sitúan en un lugar importante de la economía campesina que se ha mantenido por casi más de doscientos años. La selección natural ha hecho del cerdo criollo un animal mucho más independiente que el propio ganado criollo, con el que ha compartido por siglos las mismas sabanas [3,4]. A continuación, se presenta un breve análisis de las prácticas de manejo tradicional del cerdo criollo, su alimentación y su interés productivo en la economía colombo-venezolana.

\section{Análisis del sistema tradicional}

Dado su menor tamaño y su aparente mayor vulnerabilidad ante los predadores mencionados, el cerdo criollo tuvo que ingeniárselas con más agilidad y una mayor precocidad para sobrevivir en el nuevo medio ambiente natural del llano, en pastoreo permanente e incansable, buscando el alimento que lo caracteriza como omnívoro, con un sistema digestivo adaptado a la oferta vegetal-animal en toda su dimensión. La dieta de este cerdo criollo ha sido provista por la naturaleza, balanceada por su propia capacidad de búsqueda, selección y consumo del animal, la misma que le permite producir más de quince lechones por año.

Cuando están recién nacidas, sus camadas son de color barcino, rallados o "lapeados". La función de esta característica es la de mimetizarlos y camuflarlos como mecanismo de defensa contra los predadores en la etapa más crítica; cuando crecen y son adultos, 
esta coloración desaparece hacia las tonalidades oscuras propias de la raza sabanera (negros, pintados, azules, colorados, alazanos, canos, grifos, etc.).

Es importante destacar que los colores oscuros siempre han mostrado mayor resistencia a la insolación directa (muy frecuente en el llano), mientras que los claros resisten menos estas condiciones. Durante la etapa de lactancia, son castrados (capones) con el fin de mejorar la calidad de su carne y de eliminar la testosterona, que en el cerdo produce un fuerte olor almizclado. El descolado se aplica casi siempre en los machos capones para facilitar su identificación a mayor distancia. No se hace descolmille porque el hábito alimenticio al que deberán someterse demanda estas incisivas herramientas.

Las prácticas sanitarias se reducen a la vacunación semestral contra la peste porcina clásica y a la aplicación de algún antihelmíntico de amplio espectro para controlar formas maduras y larvarias de parásitos gastrointestinales, pulmonares y renales (Metastrongylusspp, Strongyloides, Áscaris, Ooesophagostomun, Macracanthorhynchus sp, Ascarops strongylina, Hyostrongylus rubidis, Metastrongylus elongatus y Stephanurus dentatus).

Los lechones son destetados después de tres a cuatro meses bajo el cuido materno y una bajísima mortalidad, con pesos cercanos a los $10 \mathrm{~kg}$ y a veces hasta $15 \mathrm{~kg}$. Cuando son nacidos durante la época seca, los recién destetos adquieren un nuevo régimen alimenticio a base de mango (Manguifera indica), merecure (Licania pyrifolia), caruto (Genipa americana) y otras frutas silvestres de la temporada (figura 2).

Desde el principio, han aprendido a utilizar su trompa para hozar, pero es a partir de este periodo cuando se convierte en una poderosa herramienta elongada y fuerte con funciones de azadón o pala frontal, con la que van surcando y removiendo el suelo para extraer de allí raíces, bulbos y tubérculos; lombrices de tierra (Lombricus terrestris) y otros moluscos; pequeños insectos y crustáceos; larvas y huevos de anfibios; semillas, frutas y bayas del monte, y hasta pasto fibroso y leñoso que otras especies no pueden aprovechar.

Toda esta rica oferta alimenticia es balanceada con la suplementación del suero de queso, un subproducto lácteo que está disponible en casi todas las fincas llaneras donde el principal rubro pecuario explotado es la ganadería bovina de doble propósito (carne y leche).
El suero es un recurso abundante cuya disponibilidad coincide con la parición de becerros en el sistema de sabana, que está ajustada a la época seca, y los picos altos de producción de leche coinciden con la mejor oferta forrajera de entradas de agua (inicio del periodo lluvioso). Esta integración favorable entre el subsistema bovino y el porcino permite concluir que el reloj biológico natural en condiciones de sabana está sincronizado para producir al mismo tiempo.

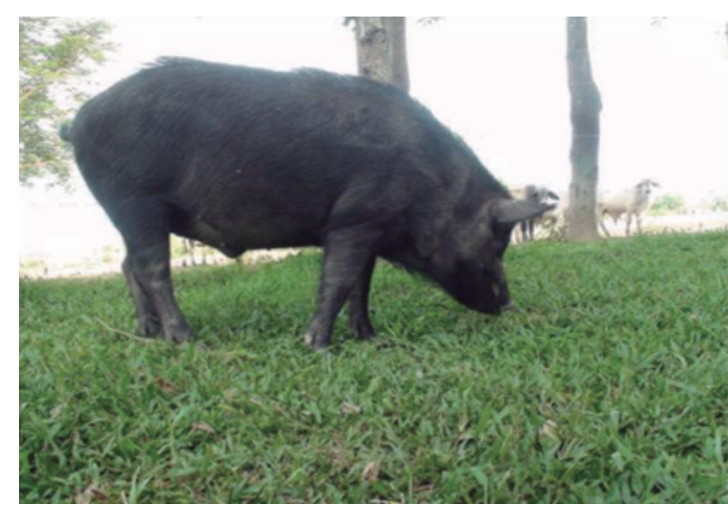

Figura 2. Un capón (cerdo castrado) exhibiendo el tuco de la cola, que muestra el manejo tradicional aplicado Fuente: Adolfo F. Cardozo, Fundo "Las Mercedes", Arauca, departamento de Arauca, Colombia

Al llegar a los 6-7 meses de edad, los adultos jóvenes deben estar pesando entre 20 y $25 \mathrm{~kg}$; a veces llegan a $30 \mathrm{~kg}$ cuando las condiciones climáticas y medioambientales lo permiten. En esta época, los capones están listos para salir al mercado.

Las cerdas jóvenes (novillas o lechonatas) que se quedan de reemplazo, con los mismos 25 kilos son servidas por el verraco, es decir que para el próximo verano (periodo de sequía) ya están dando su primer parto. Estas hembras aún son jóvenes y continúan creciendo hasta alcanzar los $50-60 \mathrm{~kg}$, cuando son llamadas madrineras por su peso, mayor edad y jerarquía en el grupo.

A primera vista, estos pesos de inicio de la edad reproductiva parecieran bajos, sobre todo si se considera un patrón de comparación con las razas industriales modernas. Hay innumerables argumentos para explicar estas apreciables diferencias, las cuales no deben ser consideradas ni mejores ni peores para uno u otro grupo genético, solo son el resultado lógico e inteligente (en términos de adaptación) para desempeñarse lo más eficientemente 
posible en las condiciones medioambientales y socioculturales propias del llano.

Esto permite estimar que el número de partos al año por cerda es de 1,5 a 2, mientras que los lechones destetos por año por cerda son cerca de 14 o 15. Si se tiene en cuenta que los costos de producción son muy bajos, se puede deducir que la rentabilidad es muy alta. No obstante, el desempeño reproductivo del cerdo criollo sabanero no ha sido estudiado, y constituye una de las principales líneas de investigación para avanzar hacia su comprensión y pleno aprovechamiento [5].

Las madrineras que alcanzan a producir otra camada en el mismo año lo hacen durante la época húmeda, es decir, iniciando el segundo semestre. Cuando los lechones son nacidos en la época húmeda, las condiciones medioambientales son menos favorables porque la oferta forrajera, aunque se ha incrementado en su biomasa, está ahora limitada en cuanto a su accesibilidad para el cerdo, debido al natural régimen de inundación cíclica de la sabana. Sin embargo, los cerditos nacen y deben ser atendidos con mayor esmero, ya que la población de ectoparásitos como moscas y gusanos es alta, igual que las enfermedades parasitarias, lo que exige intensificar las prácticas de manejo sanitario.

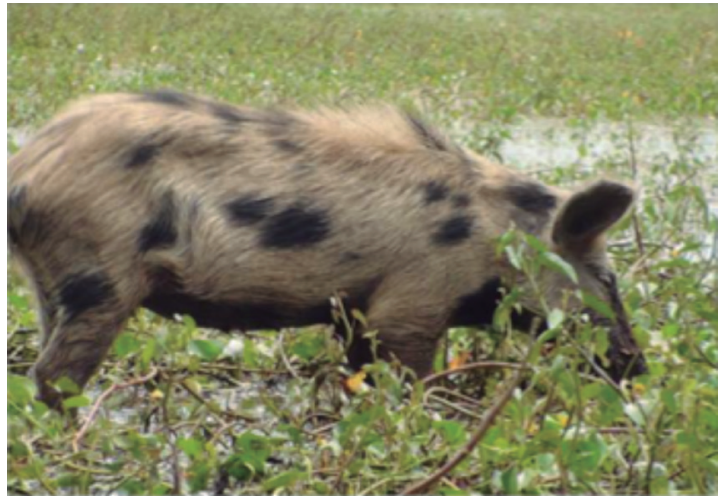

Figura 3. Una madrinera en su medio natural hozando en un boral (Euchornia crassipes)

Fuente: Adolfo F. Cardozo, Fundo "Las Bonitas", Elorza, estado Apure, Venezuela

En esta época del año, las vacas declinan la producción de leche, por lo cual el suero es más escaso y obliga a pensar en otras alternativas de suplementación, tales como el ensilaje y la suplementación con otras fuentes nutricionales, entre las que se debe incluir el alimento concentrado, en especial para las cerdas lactantes que requieren mayor cuido y atención durante las primeras semanas posparto.

A pesar de que la producción es a campo abierto, una paridera artesanal y sencilla, provista con paja y gancho de topocho en un sitio tranquilo y aislado, es ideal para que las madres den a luz, protejan y alimenten bien a sus lechones en los primeros días de vida. Cuando están más "duritos", es decir que se puedan valer por sus propios medios, ellos salen de la paridera y se integran paulatinamente al nuevo medio que los rodea. Pocos días después, la madre sale de la paridera y comienza a pastorear a campo abierto con el resto del grupo.

Recientemente, se han implementado con éxito prácticas de ensilaje de frutas como el mango (Manguifera indica) y el merecure (Licania pyrifolia), que sobrepasan la oferta aprovechable por los animales y terminan perdiéndose por la superproducción.

Las semillas de estos frutos son desechadas por los cerdos en pastoreo, que al principio solo consumen la carne o pulpa. Pero en la medida en que avanza el invierno y recrudece el clima, los cerdos vuelven a ubicarlas bajo los árboles para voltearlas y aprovecharlas, debido a que la humedad ambiental remueve el endocarpio y brota el embrión con su nueva plántula, muy rica en nutrientes que el cerdo aprovecha para su alimentación.

Otro árbol silvestre y cultivado tradicionalmente en el llano es el totumo (Crescentia sp.), el cual produce un fruto (tapara) con un mesocarpio pulposo, o "tripa", que ha sido reportado con valor forrajero para los bovinos [6]. La tripa de la tapara, que antes era desaprovechada, hoy se ofrece también en forma de ensilaje, a fin de ayudar a sostener a los porcinos durante la época crítica de julio a septiembre. Es una fuente importante de proteína y materia seca que si bien se puede ofrecer en forma directa para el consumo, es mucho más palatable en forma de ensilaje, debido al aroma que despide el alimento conservado de esta manera.

En un estudio reciente [7], se pudo demostrar que el extracto del fruto de la tapara tiene un efecto antihelmíntico en los ovejos que superó incluso el producido por los productos comerciales, lo cual hace presumir que tal vez los cerdos que consumen esta pulpa consiguen un efecto antiparasitario. El coco o cocotero (Coccos nucífera), que en las casas de la sabana se pudre en los basureros sin ningún uso, es muy útil en estos periodos de escasez, especialmente para las cerdas lactantes ya que su alto contenido en minerales y aceites vegetales son 
altamente lactopoyéticos, es decir que estimulan la producción de leche, proceso de vital importancia para la sobrevivencia de los lechones.

Una vez destetados los lechones de estas camadas "invierneras", son encaminados a los humedales más cercanos de las casas, en donde hozan para aprovechar diversas plantas acuáticas y las altas poblaciones de invertebrados como lombrices, grillos, langostas y otros insectos en estado larvario y adulto, que aportan altos niveles de proteína a la dieta del cerdo.

Los hábitos alimenticios de los cerdos en el sistema a campo abierto son variados y tienen un efecto positivo recientemente demostrado sobre la calidad de su carne $[8,9]$, lo cual explica la amplia cultura gastronómica que se ha desarrollado en el llano en torno al cerdo criollo sabanero [10].

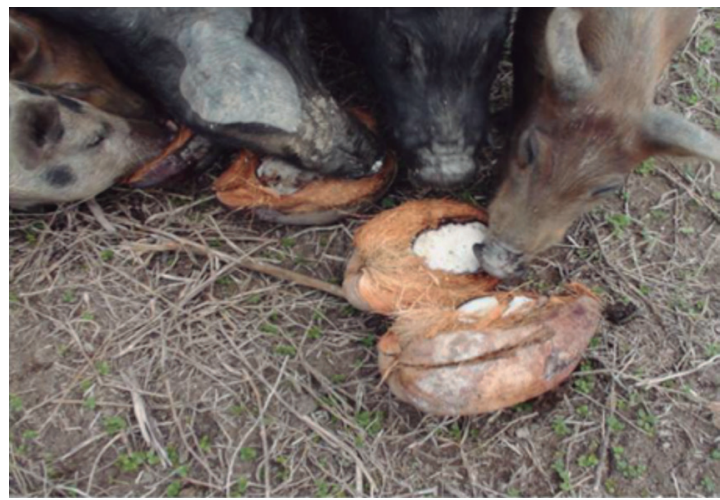

Figura 4. El grupo de marranos alimentándose de la pulpa de coco luego de haber sido abierto con un golpe de hacha

Fuente: Adolfo F. Cardozo, Fundo "Las Mercedes", Arauca, departamento de Arauca, Colombia

Tan pronto dejan de hozar en los humedales, y después de refrescarse con una zambullida, se van a husmear bajo los guayabos (Psidium guajava) y los mamones (Melicoccus bijugatus), que para esta época están de cosecha. En las matas de monte cercanas (bosques de galería), se producen otras vayas anonáceas en cosecha como la manirita (Anona manii) y la manirota (Anona purpurea). En los bancos de sabana, se encuentra también la guayabita sabanera (Psidium spp), que da frutos dos a tres veces al año durante las dos épocas. Algunos investigadores reportan que los cerdos criollos sabaneros ubican, recolectan y consumen los frutos y las semillas de 30 especies de árboles y arbustos que crecen en los diversos hábitats naturales del llano [11].
La escoba amarilla (Cytisus scoparius) es una especie de porte bajo poco consumida por los bovinos, debido a su capacidad de acumular nitratos y nitritos que son tóxicos para esta especie, pero inofensivos para el cerdo criollo. Estos consumen mucho la escoba y también otras especies de fabáceas de porte bajo como el bledo de los paraderos (Amaranthus spinosus) y la escoba brusca (Cassia tora); otras malezas de hoja ancha como el cuero de sapo o vejigón (Physalis angulata) son parte de la dieta de las tardes, antes de llegar al paradero a pastorear gramilla y flores de árboles como el madroño (Arbutus unedo).

Ya en la tarde, los cerdos se agrupan solos en la caballeriza o en la vaquera para pasar la noche, apilados en un solo montón, guarecidos del frío y la lluvia, pero alertas cuando un "brizote" llanero les avise que caerán unos cuantos mamones o guayabas.

Cuando comienzan las salidas de agua en octubre, empieza a aparecer el boro (Euchornia crassipes), uno de los alimentos más apetecidos por los cerdos que se explotan en el sistema a campo abierto en el llano. El boro es una planta propia de los ecosistemas de sabana inundable. De esta época en adelante se convierte en la dieta básica durante casi todo el verano, especialmente en los grandes esteros y humedales donde permanece el agua veranera. Esta planta, luego de florecer, acumula sus reservas de nutrientes en un bulbo subterráneo que los cerdos hozan para consumirla [11].

\section{Conclusiones}

Más de 90 familias viven en las veredas de El Matal de Flor Amarillo, y otro tanto en Monte Alto (municipio de Cravo Norte, Arauca), unas comunidades rurales ubicadas a más de 150 kilómetros de la población de Arauca. Ante toda la oferta alimenticia de productos autóctonos disponibles en una explotación porcina tradicional como la porcinocultura de los llanos colombo-venezolanos, es posible concluir que hay una oportunidad de ofertar productos orgánicos de primera calidad. Después de esta reflexión acerca del aporte del potencial del cerdo sabanero para satisfacer la demanda de mercados sofisticados y exigentes, se hace necesario desarrollar investigaciones que conduzcan a establecer parámetros productivos y reproductivos para 
mejorar la eficiencia de este sistema de explotación con una raza porcina subutilizada en el ecosistema de sabanas inundables.

\section{Referencias}

[1] Cardozo A, Rodríguez LE. ¿Por qué el cerdo criollo llanero es y debe ser pequeño y de apariencia "flaca"? Universidad de los Llanos y Universidad Cooperativa de Colombia [en prensa]; 2009.

[2] Benítez OW, Sánchez MD. Los cerdos locales en los sistemas tradicionales de producción. Estudio FAO Producción y Sanidad Animal 148 [Internet]. 2001 [citado 2015 nov 6]. Disponible en: http://www.fao. org/3/a-y2292s.pdf

[3] Martínez CG. Estado actual de los bovinos criollos y colombianos y su potencialidad en sistemas de cría (carne) y doble propósito en el ecosistema inundable. Documento presentado en: I Congreso Internacional Producción y Desarrollo Sostenible versión Sabanas Inundables. Arauca, Colombia; 2008.

[4] Rodríguez LE. Semblanza de un ganadero tradicional de las sabanas araucanas, don Pablo Canay; marzo-abril de 2008.

[5] Cardozo A, Rodríguez LE. Potencial y necesidades de investigación sobre el cerdo criollo en los llanos de Colombia y Venezuela. Revista Computadorizada de Producción Porcina. 2010;17(2).

[6] Zamora S, García Y, Bonilla G, Aguilar H, Harvey C, Muhammad I. Cómo utilizar los frutos de guanacas- te (Enterolobium cyclocarpum), guácimo (Guazuma ulmifolia), genízaro (Pithecellobium saman) y jícaro (Crescentia alata) en alimentación animal. Agroforestería en las Américas. 2001;8(31):45-9.

[7] Márquez J, Pérez E. Uso del extracto acuoso del fruto del totumo (Cressentia cujete) en el control in vitro de parásitos gastrointestinales de ovinos [trabajo de aplicación de conocimientos]; 2008.

[8] Basso L, Moisá S, Brunori J, Franco R, Bacci R, Papotto D. Calidad de la carne diferencial de cerdos producidos en sistemas al aire libre. En: Ly J, Rico C, Lon E, González C, Beldar R, Nieves D, editores. IX Encuentro de Nutrición y Producción en Animales Monogástricos. Montevideo: MGAP; 2007.

[9] Aparcicio M, Vargas J, Prieto L, Robledo J, González F, Andrada J, et al. Análisis del bienestar animal en diferentes sistemas de cría del cerdo ibérico y efecto sobre la calidad de la carne. En: Ly J, Rico C, Lon E, González C, Beldar R, Nieves D, editores. Ix Encuentro de Nutrición y Producción en Animales Monogástricos. Montevideo: MGAP; 2007.

[10] Escobar E, Cardozo A. Gastronomía tradicional del cerdo criollo producido en los llanos de Venezuela y Colombia. Ponencia del X Encuentro de Monogástricos y IV Encuentro de Cerdo Criollo. Villavicencio, Colombia; 2009.

[11] Díaz H, Cardozo A. Frutos Arbóreos Consumidos por Cerdos Criollos en Unidades Tradicionales de Producción en una Localidad del Estado Apure, Venezuela. Ponencia del x Encuentro de Monogástricos y IV Encuentro de Cerdo Criollo. Villavicencio, Colombia; 2009. 